\title{
Magneto-Hydrodynamics Flow of Newtonian Fluid over a Suddenly Accelerated Flat Plate
}

\author{
M. A. Haque \\ Department of Applied Mathematics, University of Rajshahi, Rajshahi, Bangladesh \\ Email: a_haque_amath@yahoo.com
}

How to cite this paper: Haque, M.A. (2018) Magneto-Hydrodynamics Flow of Newtonian Fluid over a Suddenly Accelerated Flat Plate. Open Journal of Fluid Dynamics, 8, 73-77.

https://doi.org/10.4236/ojfd.2018.81006

Received: January 28, 2018

Accepted: March 26, 2018

Published: March 29, 2018

Copyright $\odot 2018$ by author and Scientific Research Publishing Inc. This work is licensed under the Creative Commons Attribution International License (CC BY 4.0).

http://creativecommons.org/licenses/by/4.0/

\begin{abstract}
In this paper the laminar flow of Newtonian conducting fluid produced by a moving plate in presence of transverse magnetic field is investigated. The basic equation governing the motion of such flow is expressed in non-dimensional form. Analytic solution of the governing equation is obtained by Laplace transformation. Numerical solution of the dimensionless equation is also obtained with the help of Crank-Nicholson implicit scheme. Velocity profiles of the corresponding problem are shown in the graphs.
\end{abstract}

\section{Keywords}

Viscous Fluid, MHD Flow, Laplace Transformation, Crank-Nicholson Implicit scheme

\section{Introduction}

If a magnetic field is placed before a moving conducting fluid then the motion of the fluid is changed by the influence of the magnetic field. The magnetic field is also perturbed by the motion of the fluid: one affects the other and vice versa. The motion of the conducting fluid across the magnetic field generates electric-currents, which changes the magnetic field and the action of magnetic field on these currents gives rise to mechanical forces which modify the flow of the fluid. The electromagnetic field is governed by Maxwell's electromagnetic equations and the motion of the fluid is governed by the field equations of the fluid mechanics. In recent years, the study of MHD phenomena in liquid conductors has received considerable impetus on account of its theoretical experimental and practical applications. Schlichting [1] studied the problem of an incompressible viscous fluid flow problem produced by the oscillation of a plane solid wall. This problem is also known as stokes second problem. Von Keregek and Davis [2] performed the linear stability theory of oscillating Stokes layers. Pauton [3] ob- 
tained the transient solution for the flow due to the oscillation plane. Erdogan [4] derived the analytic solutions for the flow produced by the small oscillating wall for small and large time by Laplace transformation method. Recently Poria, Mamaloukas, layek \& Magumdar [5] derived the solution of laminar flow of viscous conduction fluid produced by the oscillating plane wall. They solved the problem both analytically and numerically in presence of magnetic field. In this paper the main aim is to investigate the effects of a transverse magnetic field on the incompressible electrically conducting fluid flow produced by a moving plate. An attempt has been made to investigate the analytic solutions for the problem. The problem has been solved numerically using well known Crank-Nicholson Implicit scheme.

\section{Formulation of the Problem}

The equation of motion of the conducting fluid in presence of transverse magnetic field is

$$
\frac{\mathrm{d} \bar{V}}{\mathrm{~d} t}=-\frac{1}{\rho} \nabla p+v \nabla^{2} \bar{V}-\frac{1}{\rho} \sigma B_{0}^{2} \bar{V}
$$

where $\bar{V}$ is the fluid velocity, $p$ the fluid pressure, $v$ the kinetic coefficient of viscosity, $B_{0}$ the uniform magnetic field, $\sigma$ the electrical conductivity.

Let us consider a flat plate extended to large distances in $x^{\prime}$ and $z^{\prime}$ directions. Again we consider an incompressible viscous fluid over a half plane solid wall $y^{\prime}=0$. Suppose the fluid is at rest at time $t^{\prime}<0$. At $t^{\prime}=0$ the plane solid wall $y^{\prime}=0$ is suddenly set in motion in $x^{\prime}$ direction at constant velocity $U_{O}$. As a result a two dimensional parallel flow will be produced near the plate. Since the fluid flows along $x^{\prime}$ direction and there is no velocity component along the direction perpendicular to the direction of flow, so the equation of conservation of mass reduces to

$$
\frac{\partial u^{\prime}}{\partial x^{\prime}}=0 .
$$

As the flow is only kept in motion by the movement of the plate, one may set the pressure gradient $\frac{\partial p^{\prime}}{\partial x^{\prime}}=0$. For unsteady case Equation (1) reduces

$$
\frac{\partial u^{\prime}}{\partial t^{\prime}}=v \frac{\partial^{2} u^{\prime}}{\partial y^{\prime 2}}-\frac{\sigma B_{0}^{2}}{\rho} u^{\prime} .
$$

Equation (2) indicates that $\mathrm{u}$ is a function of $y^{\prime}$ and $t^{\prime}$. Boundary conditions:

$$
\begin{aligned}
& u^{\prime}=0 \text { when } t^{\prime} \leq 0 \text { for all } y^{\prime}, \\
& \qquad u^{\prime}=U_{O} \text { at } y^{\prime}=0 \text { when } t^{\prime} \geq 0, \\
& u^{\prime}=0 \text { at } y^{\prime}=\infty \text { when } t^{\prime} \geq 0 .
\end{aligned}
$$

We introduce the following non-dimensional quantities

$$
y=\frac{y^{\prime}}{L}, \quad t=\frac{t^{\prime}}{T}, \quad u=\frac{u^{\prime}}{\cup_{o}},
$$


where $L$ and $T$ represent the characteristic length and characteristic time respectively. Setting these non-dimensional quantities in Equation (3), we get

$$
\frac{\partial u}{\partial t}=\frac{\partial^{2} u}{\partial y^{2}}-M^{2} u,
$$

where

$$
\begin{gathered}
\rho v=\mu, \\
M^{2}=B_{0} L \sqrt{\frac{\sigma}{\mu}} .
\end{gathered}
$$

Here the number $M$ is a non-dimensional number and is called Hartmann number.

In this case the boundary conditions may be written as

$$
\begin{aligned}
& t \leq 0: u(y, 0)=0, \quad \text { for all } y \\
& t \geq 0: u(0, t)=1, \quad u(\infty, t)=0
\end{aligned}
$$

\subsection{Analytic Solution}

We introduce the Laplace transformation and inverse Laplace transformation as

$$
L\{u(y, t)\}=\bigcup(y, s)
$$

and

$$
L^{-1}\{\mathrm{U}(y, s)\}=u(y, t) .
$$

We have

$$
L\left\{\frac{\partial U}{\partial t}\right\}=s L\{u\}-u(y, o)=s U
$$

and

$$
L\left\{\frac{\partial^{2} u}{\partial y^{2}}\right\}=\frac{\mathrm{d}^{2}}{\mathrm{~d} y^{2}}[L\{u\}]=\frac{\mathrm{d}^{2} \cup}{\mathrm{d} y^{2}} .
$$

From Equation (5), we have

$$
\frac{\mathrm{d}^{2} U}{\mathrm{~d} y^{2}}-\left(s+M^{2}\right) \cup=0 .
$$

With the help of boundary condition (6), we get

$$
U(o, s)=L\{u(0, t)\}=\frac{1}{s} .
$$

The Solution of Equation (11) is

$$
\mathrm{U}(y, s)=c_{1} \mathrm{e}^{y \sqrt{s+M^{2}}}+c_{2} \mathrm{e}^{-y \sqrt{s+M^{2}}} .
$$

Since $u$ is finite for $y \rightarrow \infty$, we must have $c_{1}=0$.

Equation (13) reduces to

$$
U(y, s)=c_{2} \mathrm{e}^{-y \sqrt{s+M^{2}}}
$$




$$
\therefore \cup(0, s)=c_{2} \Rightarrow c_{2}=\frac{1}{s} \text {. }
$$

Thus the Equation (13) reduces to

$$
U(y, s)=\frac{1}{s} \mathrm{e}^{-y \sqrt{s+M^{2}}} .
$$

Taking inverse Laplace transformation of Equation (12), we have

$$
u(y, t)=-\frac{1}{\pi} \int_{-M^{2}}^{\infty} \frac{\mathrm{e}^{-\left(u+M^{2}\right) t}}{u+M^{2}} \sin (y \sqrt{u}) \mathrm{d} u
$$

\subsection{Numerical Solution}

The Equation (5) with initial and boundary conditions (6) is solved by finite difference technique. The crank-Nicholson implicit scheme is used to solve the parabolic type of equation. In this scheme, the time derivative term is represented by forward difference formula while the space derivative term is represented by the average central difference formula. To do this the temporal first derivative can be approximated by

$$
\frac{\partial u}{\partial t} \approx \frac{u_{i}^{l+1}-u_{i}^{l}}{\Delta \tau}
$$

The second derivative in space can be determined at the midpoint by the averaging the difference approximations at the beginning $\left(t^{l}\right)$ and at the end $\left(t^{l+1}\right)$ of the time increment as

$$
\frac{\partial^{2} u}{\partial y^{2}} \cong \frac{1}{2}\left[\frac{u_{i+1}^{l}-2 u_{i}^{l}+u_{i-1}^{l}}{(\Delta \eta)^{2}}+\frac{u_{i+1}^{l+1}-2 u_{i}^{l+1}+u_{i-1}^{l+1}}{(\Delta \eta)^{2}}\right]
$$

Substituting Equation (17) and Equation (18) into Equation (5), we get

$$
\frac{u_{i}^{l+1}-u_{i}^{l}}{\Delta \tau}=\frac{1}{2}\left[\frac{u_{i+1}^{l}-2 u_{i}^{l}+u_{i-1}^{l}}{(\Delta \eta)^{2}}+\frac{u_{i+1}^{l+1}-2 u_{i}^{l+1}+u_{i-1}^{l+1}}{(\Delta \eta)^{2}}\right]-\frac{M^{2}}{2}\left(u_{i}^{l+1}+u_{i}^{l}\right)
$$

or

$$
r u_{i-1}^{l+1}-(2 r+s+1) u_{i}^{l+1}+r u_{i-1}^{l+1}=(2 r-1+s) u_{i}^{l}-r\left(u_{i-1}^{l}+u_{i+1}^{l}\right)
$$

where

$$
r=\frac{\Delta \tau}{2(\Delta \eta)^{2}}, \quad s=\frac{\Delta \tau M^{2}}{2}
$$

The Equation (20) may be written as

$$
-r u_{i-1}^{l+1}+k_{1} u_{i}^{l+1}-r u_{i-1}^{l+1}=r\left(u_{i-1}^{l}+u_{i+1}^{l}\right)+k_{2} u_{i}^{l}
$$

where $k_{1}=1+2 r+s$ and $k_{2}=1-2 r-s$.

The system of algebraic equations in tri-diagonal form is solved by Thomas algorithm for each time level. In this problem some grid points have been consider for numerical computation. $\mathrm{u}$ is obtained at each grid point at each time interval. The Figure 1 below is drawn for various values of y when $t=0.4$. 


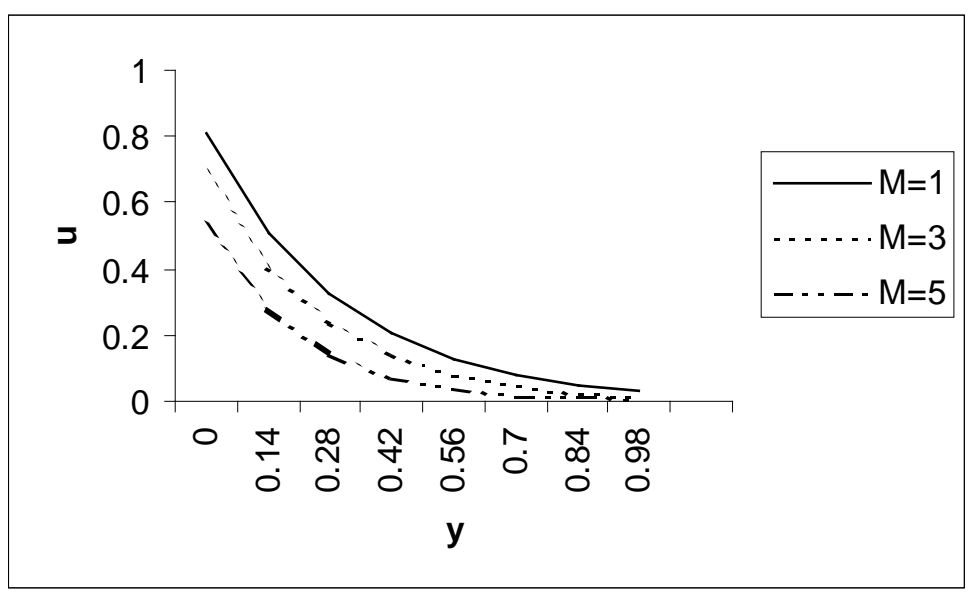

Figure 1. Velocity profile for different values of Hartmann number M.

\section{Results and Discussion}

Numerical results are displayed by the above figure. This figure shows that the velocity of the fluid decreases as the magnetic field increases. The velocity decreases gradually and attains almost zero velocity at a sufficient large distance from the plate.

\section{References}

[1] Schlichting, H. (1968) Boundary Layer Theory. 6th Edition, MacGraw-Hill, New York.

[2] Kerezek, V.C. and Davis, S.H. (1974) Linear Stability Theory of Oscillation Stokes Layers. Journal of Fluid Mechanics, 62, 753-773.

https://doi.org/10.1017/S0022112074000929

[3] Pauton, R. (1968) The Transients for Stokes's Oscillation Plane a Soliution in Terms of Tabulated Functions. Journal of Fluid Mechanics, 31, 819. https://doi.org/10.1017/S0022112068000509

[4] Erdogon, M.E. (2000) A Note on an Unsteady Flow of a Viscous Fluid Due to an Oscillating Plane Wall. The International Journal of Non-Linear Mechanics, 35, 1-6. https://doi.org/10.1016/S0020-7462(99)00019-0

[5] Poria, S., Mamaloukas, C., Layek, G.C. and Mazumdar, H.P. (2001) Some Effects of a Transverse Magnetic Field on the Flow of a Viscous Conducting Fluid Produced by an Oscillating Plane Wall. Journal of Technical Physics, Warszawa. 